\title{
A study on laser touch dressing of electroplated diamond wheels using pulsed picosecond laser sources
}

\section{Report}

Author(s):

Dold, C.; Transchel, R.; Rabiey, Mohammad; Langenstein, Peter; Jaeger, C.; Pude, Frank; Kuster, K.; Wegener, Konrad

Publication date:

2012

Permanent link:

https://doi.org/10.3929/ethz-a-007577830

Rights / license:

$\underline{\text { In Copyright - Non-Commercial Use Permitted }}$ 
A study on laser touch dressing of electroplated diamond wheels using pulsed picosecond laser sources

\author{
C.Dold ${ }^{1}$, R. Transchel ${ }^{1}$, M. Rabiey ${ }^{1}$, P. Langenstein ${ }^{3}$, C. Jaeger ${ }^{3}$, F. Pude ${ }^{1}$, F. Kuster ${ }^{2}$, K. Wegener ${ }^{1,2}$ \\ ${ }^{1}$ inspire AG, ETH, Zurich, Switzerland - ${ }^{2}$ Institute of Machine Tools and Manufacturing (IWF), ETH, Zurich \\ Switzerland - ${ }^{3}$ Faessler AG, Dubendorf, Switzerland
}

Submitted by J.-P. van Griethuysen (1), Yverdon-les-Bains, Switzerland

\begin{abstract}
An investigation to explore the possibility of touch dressing of electroplated diamond wheels using a pulsed picosecond laser (ps-laser) beam (Yb:YAG) is presented. The laser beam is irradiated on the wheel to cut the diamond grains within a defined grain protrusion without any thermal damage on the nickel bond. The laser parameters were systematically varied to achieve a reliable touch dressing process. The microscopic investigations prove thermal damages neither on the diamond grain nor on the bond. Series of $\mathrm{SiC}$ wheel dressing experiments were carried out to compare mechanical touch dressing of the electroplated diamond wheel with laser dressed wheels.
\end{abstract}

Keywords:

Grinding, Super abrasive, Laser Truing

\section{INTRODUCTION}

Touch dressing of superabrasive wheels is usually applied for an electroplated bond material, where an accurate profile and defined grain protrusion is necessary. Touch dressing of diamond or CBN wheels can be conventionally done using a diamond roll or wheel as truer and/or dresser. In the case of touch dressing, both the wheel and truer are diamond plated, and have almost the same hardness resulting in a time consuming and relatively expensive process. That is why an alternative method to reduce process time as well as cost is a matter of high interest in industry.

Upon discovering the benefit of lasers in the machining industry, laser conditioning has always been an interesting alternative to conventional mechanical dressing. However, due to certain drawbacks and technological problems, its use in industrial applications was limited. In the case of touch dressing of electroplated diamond wheels, it is much more difficult to reach the realized target because the laser beam must cut the diamond grains with high accuracy, without any kind of thermal damage either to the diamond grain (graphitization, residual stress, etc.) or to the nickel bond material. This research is an important step forward to the realized target using a pulsed ps-laser. This paper presents a successful attempt on touch dressing of an electroplated diamond wheel using a ps-laser source.

\section{LASER DRESSING, STATE OF THE ART}

Laser technology for conditioning of superabrasives was first proposed by Westkaemper [1] for dressing and truing of resin bonded CBN grinding wheels. The results show a topography with sufficient chip clearance compared with conventional dressing methods. It also illustrated approximately the same values for grinding force and roughness after grinding. Timmer [2] utilized a Nd:YAG laser to differently bonded diamond and $\mathrm{CBN}$ grinding wheels both radially and tangentially to the wheel surface. $\mathrm{He}$ also proposed a model for laser conditioning based on thermal and optical characteristics of the system. Kang et al [3] conducted a study of truing of resin and metal-bonded diamond wheels by utilizing pulsed Nd:YAG laser radiation tangentially to the grinding wheel surface. It was observed that the resin bonding material started to decompose, the bronze bonding material either melted or vaporized, and the diamond grains that are slightly damaged were removed with respect to the sputtering of the bond materials. Yung et al [4] used an acousto-optically Q-switched Nd:YAG laser to dress a resin bonded CBN wheel. A $10-15 \%$ reduction in grinding force compared to conventional dressing as well as good topography of the wheel without destroying or damaging the CBN grains was reported. Furthermore, the Q-switched laser showed a lower heat accumulation on the wheel surface than continuous wave laser processing. Wang et al [5] developed a model to predict various parameters such as incident beam angle, focal offset and incident power during laser truing of vitrified and metal bonded CBN grinding wheels. For the resin bonded $\mathrm{CBN}$ wheel, a numerical simulation was developed by Xie et al [6] to investigate and characterize the topography of the grinding wheel after laser dressing. Hosokawa [7] used Nd:YAG laser radiation also and reported successful dressing of metal bonded wheels. By coordinating the laser parameters and wheel rotation speed, the appropriate grain protrusion height can be generated. Detectable damage to diamond particles, such as micro-cracks or graphitization, are not observed below a critical laser power setting. Kunidea et al [8] used third harmonic generation of the Nd:YAG laser for conditioning ultrafine resin bonded diamond wheels. This resulted in higher cutting edge densities than one treated using conventional conditioning with the cap truer method. Chen et al [9] used an acousto-optically Q-Switched $\mathrm{Nd}$ :YAG pulsed laser for the conditioning of bronze bonded diamond wheels. The result shows that pulsed $\mathrm{Nd}$ :YAG lasers are more suitable than continuous wave (CW) lasers.

Based on the literature survey, a patent was found regarding touch dressing with a laser system [10]. But no direct investigation that shows the advantage on laser touch dressing of diamond grinding wheels was carried out.

\section{OBJECTIVES AND EXPERIMENT CONDITION}

\subsection{Laser system}

The experimental setup (Fig. 1) consists of the laser system (1) producing laser pulses with a pulse width $t_{P}<10 \mathrm{ps}$ and a maximum pulse energy of $\mathrm{e}_{\mathrm{P}}=125 \mu \mathrm{J}$ at a maximum repetition rate of $f_{P}=400 \mathrm{kHz}$. The experimental wavelength is $\lambda=1030 \mathrm{~nm}$ in the near infrared. The setup 
consists of multiple mirrors, a beam expansion unit $(4,5)$ and a two-dimensional scanning system (8). The beam expansion unit itself consists of a concave and a convex lens and increases the beam diameter $\mathrm{d}_{\mathrm{B}}=5 \mathrm{~mm}$ to $\mathrm{d}_{\mathrm{B}}=8.5$ $\mathrm{mm}$ prior to entering the scanhead. This results in a focal diameter of $\mathrm{d}_{\mathrm{B}, \text { foc }}=22 \mu \mathrm{m}$ on the workpiece.

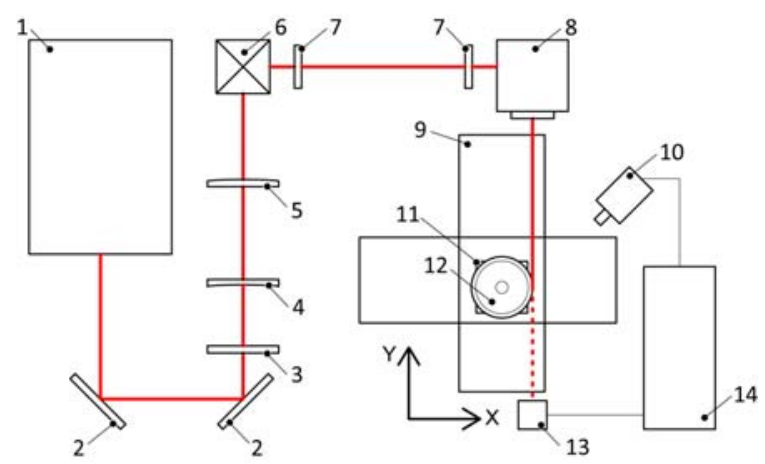

Fig. 1. Experimental setup of the system. (1) Laser system, (2) mirrors, (3) $\lambda / 2$ plate, (4) plano-concave lens, (5) planoconvex lens, (6) periscope, (7) iris diaphragms, (8) Y,Z scanning unit, (9) X,Y motorized translation stage, (10) online camera, (11) C-axis rotary stage, (12) specimen, (13) power meter, (14) data acquisition unit.

The sample piece (12) is mounted on a CNC controlled three-axis-system which consists of a X- and Y-axis and also a $\mathrm{C}$-axis which allows for rotation around the $\mathrm{Z}$-axis. The focal position can be varied by traveling in the Ydirection. The work sample is attached to the rotating $\mathrm{C}$ axis.

\subsection{Laser test condition, design of experiments}

The experiments are subdivided into two stages. First a full factorial design is implemented to find process boundary parameters to execute a second stage of statistical experiments. Variation parameters are laser power, scanning speed of the galvanometer mirrors, the pulse repetition rate and the number of repetitions for each processing line. The second stage was a statistical experiment series using a design-of-experiments (DoE) approach. Both experimental series are executed on flat samples which consist of exactly the same structure as the electroplated wheel. The diamond grains are embedded in a uniformly thick layer of exactly the same bonding material as the final cylindrical samples. Validation experiments with the found parameters are conducted on the cylindrical samples. Therefore the cylindrical sample surface was subdivided into twelve sections. Each section is marked with an engraved rectangle to do comparison measurements before and after sample processing. Single grain studies are therefore possible as well as Scanning Electron Microscopy (SEM) and Raman spectroscopy comparisons before and after laser irradiation.

For the execution of the full factorial design the average laser power is varied between $\mathrm{P}_{\text {avg }}=4.4-48.4 \mathrm{~W}$, pulse repetition rate between $f_{P}=25-400 \mathrm{kHz}$, and processing speed between $v_{f}=100-1900 \mathrm{~mm} / \mathrm{s}$. The amount of repetitions is kept constant at ten repetitions for each experiment. In order to reduce the necessary amount of experiments and to find the parameters which are most influential on the processing results, a statistical approach in form of DoE by use of the Taguchi-method is applied. The four experimental parameters are the pulse repetition rate, laser power, processing speed, and the number of repetitions for each line. These parameters are physically independent, so the Analysis Of Variance (ANOVA) can be applied. The experimental series consist of nine experiments per series execution (L9 design). The goal is to find the optimum levels of process control parameters in order to achieve robust and effective process conditions.

Following these experiments, a number of validation experiments are carried out. These included generating surface structured samples with found processing parameters and analyzing those with Raman- spectroscopy, SEM imaging, Energy Dispersive X-ray spectroscopy (EDX) analysis as well as three-dimensional measurements to evaluate the processed grain topography on the sample.

\subsection{Test condition}

Dressing experiments were carried out using laser and mechanical touch dressed electroplated diamond wheels by dressing of a $\mathrm{SiC}$ grinding wheel to analyze and compare dressing forces as well as wear progression. The touchdressed diamond wheels underwent dressing operations at a universal cylindrical grinding machine (Studer S31). Test tools were attached to a high frequency spindle (Fischer MFM-1224/42 HSK-C40) with high stiffness in radial direction $(\mathrm{k}=140 \mathrm{~N} / \mu \mathrm{m})$. A vitrified bonded silicon carbide ( $\mathrm{SiC}$ ) grinding wheel is employed to be dressed (Cerfine $\mathrm{C}$ 120/3 P4). A Rotating Component Dynamometer (RCD Kistler Winterthur-Switzerland, type: Z15168, SN: 473735) is mounted on the work piece spindle, where the grinding wheel is clamped to measure the tangential force $F_{t}$ and normal force $\mathrm{F}_{\mathrm{N}}$ during the dressing operation. Thus, the dressing kinematics correspond to dressing operations with rotating tools and an additional radial feed applied. Oil (Blaser - grinding oil HC 5) is used as a cooling lubricant. The dressing parameters are stated in Table 1 . in which $v_{\text {fr }}$ represents radial feed rate $(\mathrm{mm} / \mathrm{min}), \mathrm{v}_{\mathrm{w}}$ is the workpiece speed $(\mathrm{m} / \mathrm{s}), \mathrm{q}$ is the speed ratio and $\mathrm{Q}^{\prime}{ }_{\mathrm{w}}$ is the specific material removal rate $\left(\mathrm{mm}^{3} / \mathrm{mm} . \mathrm{s}\right)$.

Table 1. Dressing parameters for the wear analysis.

\begin{tabular}{|c|c|c|c|c|}
\hline Parameter & $\mathbf{v}_{\text {fr }}$ & $\mathbf{v}_{\mathbf{w}}$ & $\mathbf{q}$ & $\mathbf{Q}_{\mathbf{w}}{ }^{\prime}$ \\
\hline Value & 0.15 & 1.5 & 0.75 & 1.5 \\
\hline
\end{tabular}

The dressing forces are measured at regular intervals with respect to wear progression. Force signals are detected by the RCD and broadcasted wirelessly to the charge amplifier via a telemetry sensor. The sample rate is set at a frequency of $\mathrm{f}_{\text {sample }}=6 \mathrm{kHz}$, due to the comparably low cutting speed $\mathrm{v}_{\mathrm{c}}=2 \mathrm{~m} / \mathrm{s}$, and recorded with commercially available measurement software (LabView).

\subsection{Measurement condition}

The twelve reference areas $(2 \mathrm{~mm} \times 2 \mathrm{~mm})$ were equidistantly, circumferentially distributed on the abrasive layer (Fig. 2). The analysis of the topography is realized by adapting an evaluation line which is adjusted by means of distinctive points on the bonding matrix in relation to the laser-induced reference area. This procedure enables the precise repeatability of the evaluation line adaption before and after the dressing process. The measured topography data is averaged over the twelve reference areas to obtain a 
topography description of each test tool. Additionally, data of the bearing area fraction is used to generate equivalent bearing area fraction curves to be able to compare the wear progression of the test tools.
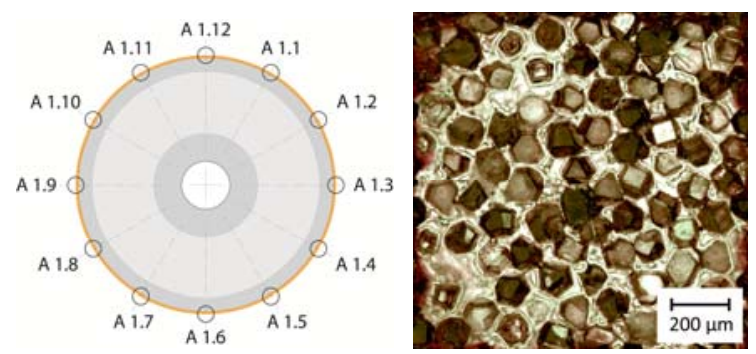

Fig. 2. Circumferentially distributed reference areas (left), microscope image of a reference area (right).

\section{EXPERIMENTAL RESULTS}

Evaluation of multiple validation samples in terms of raman-spectroscopy (Fig. 3) reveals that within the cut kerf, the presence of a graphite layer is inexistent. Even after irradiation with ten repetitions and multiple pulses per area, the measured peak position value is $1332.49 \mathrm{~cm}^{-1}$ compared to the expected value which is $1,332.5 \mathrm{~cm}^{-1}$. This is in good agreement to the quality of natural or High-Pressure HighTemperature (HPHT) synthetic diamond [11]. There is no evidence of a second peak around the graphite peak position $\left(1580 \mathrm{~cm}^{-1}\right)$.

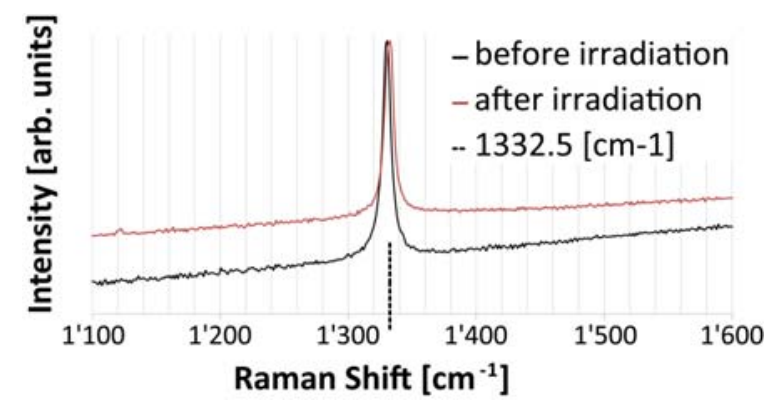

Fig. 3. Raman-spectra of industry-type IIa diamond before and after laser irradiation $\left(\mathrm{t}_{\mathrm{P}}=10 \mathrm{ps},, \lambda=1030 \mathrm{~nm}, \mathrm{~F}=\right.$ $22.82 \mathrm{~J} \mathrm{~cm}^{-2}, \mathrm{f}_{\mathrm{P}}=400 \mathrm{kHz}, \mathrm{v}_{\mathrm{f}}=700 \mathrm{~mm} / \mathrm{s}, 10 \mathrm{rep}$.).

In order to evaluate the geometrical surface properties, three-dimensional measurement images are taken, and a section profile through a machined and an unmachined area on a cylindrical sample is analyzed.

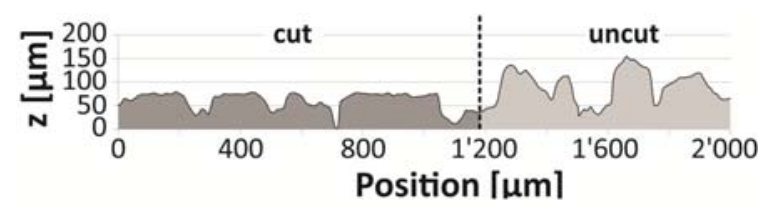

Fig. 4. Cross section of a cut and an uncut section after tangential laser irradiation of the left part of the sample $\left(t_{\mathrm{P}}=10 \mathrm{ps},, \lambda=1030 \mathrm{~nm}, \mathrm{~F}=25.79 \mathrm{~J} \mathrm{~cm}^{-2}, \mathrm{f}_{\mathrm{P}}=400 \mathrm{kHz}\right.$, 300 rep.).

As it can be seen in (Fig. 4), the generated surface is cleanly cut over the entire surface. A detailed analysis of the surface structure is discussed including the dressing results. Scanning electron microscopy measurements are conducted on a radially irradiated flat sample in order to evaluate cut quality and bonding material effects (Fig. 5). During these experiments, the laser beam processed a line geometry over the sample with 300 repetitions. As a result, the grain is completely cut into two separate pieces. Two comparison images are shown of the same grain on the sample. The irradiated grain shows a clean cut through the diamond grain as well as redeposited bonding and base substrate material as can be seen via an EDX analysis (Fig. 6).
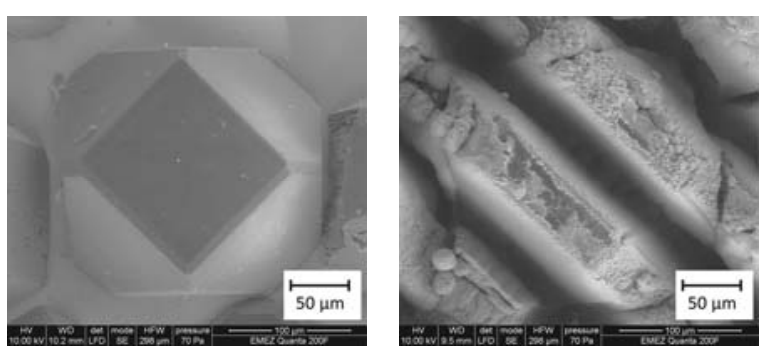

Fig. 5. SEM images of diamond grain before (left) and after radial laser irradiation (right) with bonding and substrate material redeposited on the grain surface $\left(t_{\mathrm{p}}=10 \mathrm{ps}, \lambda=\right.$ $1030 \mathrm{~nm}, \mathrm{e}_{\mathrm{P}}=99 \mu \mathrm{J}, \mathrm{f}_{\mathrm{P}}=400 \mathrm{kHz}, 300 \mathrm{rep}$.).

This is done on a small area on top of the grain right next to the cut kerf within the grain. There is no visible evidence of cracks or other damages within the grain itself.

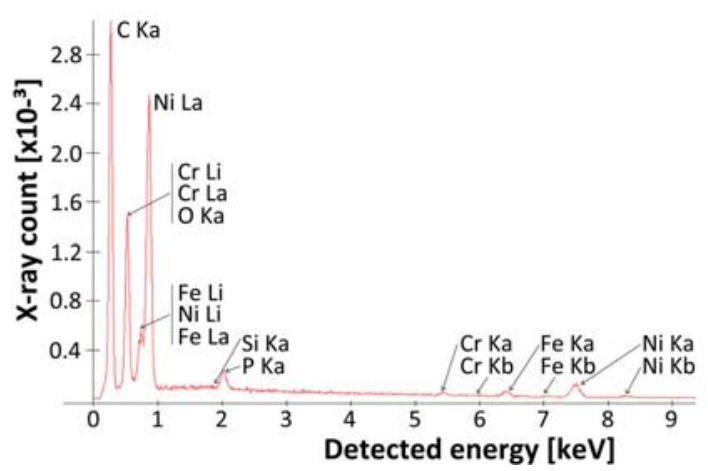

Fig. 6. EDX image of the cut kerf side of a laser irradiated diamond grain.

Within the EDX image of the sample, there are clear peaks at the carbon-line (D-line) which is typical for the diamond structure. This is followed by nickel, chromium, oxygen as well as iron. The bond material consists of nickel and the substrate material was stainless steel which gives the peaks at iron. The oxygen peak is existent, since the experiments are conducted in air as ambient gas. In order to reduce this effect nitrogen or argon as ambient gas could be used. In EDX-studies of the diamond, the bonding material and the base material alone, it can be shown that all material components shown on the graph in Fig. 6 are independently evident before laser irradiation. The dressing forces were measured in irregular intervals of the entire removal process. As can be seen in Fig. 7, the dressing forces of laser touch dressed diamond wheels are considerably lower compared to mechanical touch dressed diamond wheels. The mechanically touch dressed test tool obviously obtains almost stable progression of the normal force with a specific material removal $\mathrm{V}^{\prime}{ }_{\mathrm{w}}$ of approx. $5000 \mathrm{~mm}^{3} / \mathrm{mm}$. This effect is caused by gradual flattening of the abrasive layer. 
However, the laser touch dressed tool obtains an improved material removal rate due to decreased process forces.

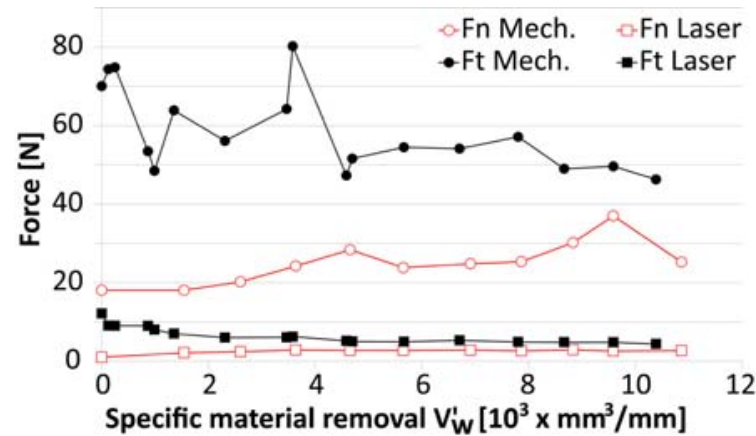

Fig. 7. Normal and tangential forces of laser touch dressed and mechanical touch dressed diamond wheels.

The topography of the dressing wheel is measured by the Alicona IFM G4 microscope and the values are determined using the Alicona evaluation software. Data of the bearing area fraction curve is averaged and used to generate Substitute-Abbott-Firestone-Curves (SAFC). These substitute SAFCs are used to compare the topography of mechanical touch dressed and laser touch dressed electroplated wheels. Fig. 8 displays the SAFC prior to the touch dressing process (upper curves) as well as the alteration of the bearing area fraction after touch dressing (middle curves) and the dressing process (lower curves).

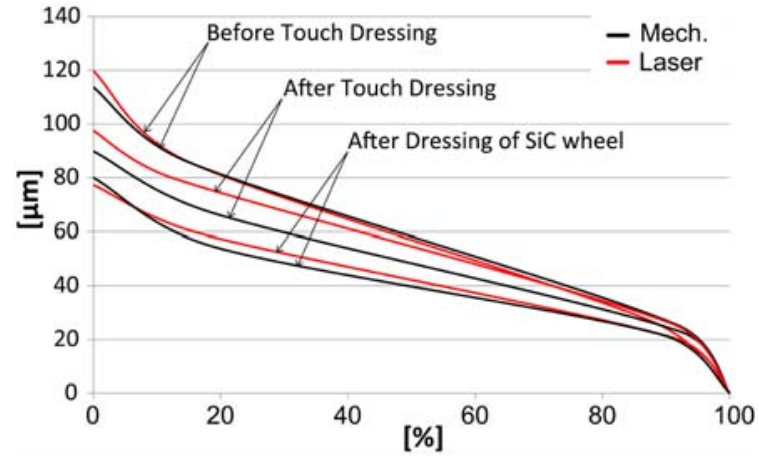

Fig. 8. Comparison of the Abbott-Firestone-Curves before and after laser touch dressing, and the dressing process.

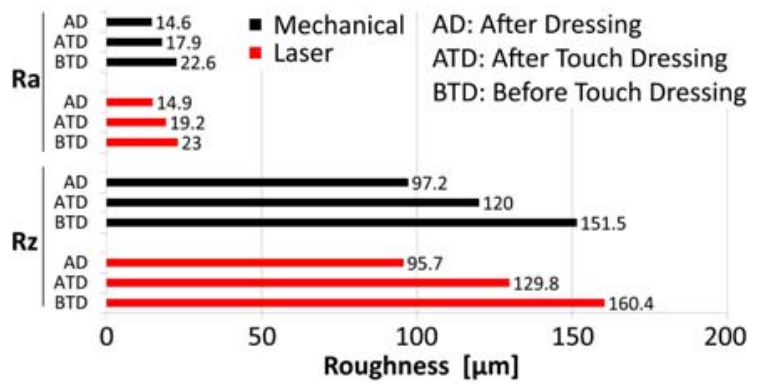

Fig. 9. Comparison of $\mathrm{Ra}$ and Rz-roughness values before and after touch dressing and after the $\mathrm{SiC}$ dressing process.

Apparently, the wear characteristic of mechanical touch dressed tools is slightly better than laser touch dressed tools. Though, the two lower SAFC's overlap at approx. 10\% of the material fraction which leads to the assumption of different wear mechanisms. A roughness comparison ( $\mathrm{Ra}$, $\mathrm{Rz}$ ) of mechanical and laser touch dressed tools shows a slight advantage in favour of mechanical touch dressed diamond wheels (Fig. 9).

\section{CONCLUSIONS \& NEXT STEPS}

By using ps-laser sources, a great potential for high precision and a highly reliable method for touch dressing of the diamond grinding wheels is observed. Diamond grains can be cut without remarkable graphitization effects. Comparison of wheel topography before and after mechanical and laser touch dressing shows a very similar form within Abbott-Firestone-curves as conventional roughness values ( $\mathrm{Ra}$ and $\mathrm{Rz}$ ). The lower dressing forces after dressing of $\mathrm{SiC}$ grinding wheels with the same dressing parameters prove higher dressing efficiency in terms of grinding forces and specific energy. Generally an effective and reliable laser touch dressing process of electroplated diamond wheels can be achieved using a well defined parameter set together with a ps-laser source. One possible application field is diamond touch dressing processes used for gear manufacturing. This will be the next immediate step of this research work.

\section{ACKNOWLEDGMENTS}

The authors wish to gratefully acknowledge the financial support which was granted by the Swiss Innovation Promotion Agency as well as the technical support provided by Faessler AG, Dubendorf and Trumpf Laser AG, Baar, both located in Switzerland.

\section{REFERENCES}

[1] Westkaemper E (1995) Grinding assisted by Nd:YAG Laser, Annals of the CIRP, $44: 317-320$.

[2] Timmer JH (2001) Laserkonditionieren von CBN- und DiamantSchleifscheiben. Dissertation, Braunschweig university.

[3] Kang RL, Yuan JT, Zhang YP, Ren JX (2001) Truing of diamond wheels by Laser, Key Engineering Materials, 202-203:137-142.

[4] Yung KC, Chen GY, Li LJ (2003) The laser dressing of resinbonded CBN wheels by a Q-switched Nd:YAG laser, Int. J. of advanced Manufacturing, 22:541-546.

[5] Wang XJ, Wang RK, Xu WJ, Wang LJ, Guo DM (2007) Modeling of laser dressing for metal-bond diamond grinding wheel, Key Engineering Materials, 329:145-150.

[6] Xie XZ, Chen GY, Li LJ (2004) Dressing of resin-bonded superabrasive grinding wheels by means of acousto-optic Q-switched pulsed Nd:YAG laser, Optics \& Laser Technology, 36/5:409-419.

[7] Hosokawa A, Ueda T, Yunoki T (2006) Laser dressing of metal bonded diamond wheel, Annals of the CIRP, 55/1:329-332.

[8] Kunidea Y, Matsuura H, Kodama S, Yoshihara N, Yan J, Kuriyagawa T (2007) Development of a new laser conditioning method for ultra-fine grit diamond wheels, Key Engineering Materials, 329:175-180.

[9] Chen G, Mei L, Zhang B, Yu C, Shun K (2010) Experiment and numerical simulation study on laser truing and dressing of bronzebonded diamond wheel, Optics and Lasers in Engineering, 48/3:295304.

[10] Tomoaki N, Takashi A, Masahiko H, Yusaku M (2007) Dress gear, and manufacturing method and apparatus therefore. Pat. JP $2007-$ 276034

[11] Linares R, Doering P (1998) Properties of large single crystal diamond, Diamond and related materials. 8:909-915. 